\title{
Interaction of aluminium with hydrogen in twinning-induced plasticity steel
}

\author{
Eun Ju Song ${ }^{a}$ H. K. D. H. Bhadeshia ${ }^{\mathrm{a}, \mathrm{b}}$ Dong-Woo Suh ${ }^{\mathrm{a}}$ \\ ${ }^{a}$ Graduate Institute of Ferrous Technology, Pohang University of Science and \\ Technology, Pohang 790-784, Republic of Korea \\ b Materials Science and Metallurgy, University of Cambridge, CB2 3QZ, U.K.
}

\begin{abstract}
Alloying with aluminium can mitigate the hydrogen embrittlement of twinninginduced plasticity steels. First-principles calculations indicate a new mechanism for this observation, that aluminium atoms are weak traps for hydrogen, because of a local dilation around them. This in turn allows the aluminium-containing steel to absorb more hydrogen, as confirmed by previously unexplained observations in the literature. Calculations using the Oriani theory show that aluminium can retard the diffusion of hydrogen in twinning-induced plasticity steel via the atomic trapping mechanism.
\end{abstract}

Key words: hydrogen trapping, hydrogen thermal desorption, TWIP, first-principles calculation

It is established that high-manganese twinning-induced plasticity (TWIP) steels exhibit combinations of elongation and strength that are attractive for many potential applications, but particularly in the automotive industries [13]. However, some variants of TWIP alloys are susceptible to hydrogen-induced failure [4-6], but it is known that adding less than $2 \mathrm{wt} \%$ of aluminium ameliorates the situation [7]. A number of mechanisms have been proposed to explain the role of aluminium:

- It has been suggested that aluminium suppresses strain aging caused by carbon, thus reducing the flow stress and hence making hydrogen embrittlement less likely [8].

- The aluminium has been postulated to reduce the absorption of hydrogen $[9,10]$ but experimental observations contradict this - a variety of thermal desorption spectroscopy suggests that the amount of hydrogen absorbed in the aluminium-containing steel is greater than in the alloys without aluminium $[11,12]$, observations that are as yet unexplained. 
- One interpretation builds on the effect of aluminium in increasing the stacking fault energy, thus reducing the possibility of transformation into $\varepsilon$ martensite or to twin and thereby mitigating hydrogen effects since twin and martensite interfaces feature at fracture surfaces [11].

- Another idea based on nanoindentation measurements is that the influence of hydrogen in reducing the shear modulus of the austenite contributes to embrittlement via the HELP mechanism; the presence of aluminium reduces the effect of hydrogen on the modulus and hence on the degree of embrittlement [12].

It is possible that all of these mechanisms actually play a role, but there recently has been an inspiring publication based on first principles calculations of binding energies in ferrite [13]. That work indicated that aluminium-alloyed ferrite should be more resistant to hydrogen than silicon-alloyed ferrite. It was therefore decided to investigate whether similar calculations can reveal the possible role of aluminium in TWIP steels. As will be seen later, there are some fascinating outcomes which tally with previously unexplained experimental observations.

The calculations were performed using the Vienna Ab Initio Simulation Package [14-16] within the generalised gradient approximation of the PerdewBurke-Ernzerhof (PBE) form [17] for electron exchange and correlation. The austenite was simulated using a $2 \times 2 \times 2$ supercell of the face-centred cubic (fcc) structure and calculations of the bulk properties were performed with a sufficiently high plane-wave cutoff energy, $400 \mathrm{eV}$. A hydrogen atom was added in both pure austenite, $\mathrm{Fe}_{32}$ and that containing $\mathrm{Al}, \mathrm{Fe}_{31} \mathrm{Al}$. $\mathrm{A} 7 \times 7 \times 7 k$-point Monkhorst-Pack grid was used to sample the Brillouin zone. The first-order Methfessel-Paxton method [18] was used for the Fermi-surface smearing in order to obtain accurate forces, with a smearing width of $0.1 \mathrm{eV}$. Both cell shape and atomic positions were permitted to relax for bulk calculations.

Austenite at ambient temperature is paramagnetic but its ground state has an antiferromagnetic double layer (AFMD) structure [19]. However AFMD is not consistent with the paramagnetic state and its relaxed lattice structure is tetragonal, $a=3.54 \AA, c=3.75 \AA$, [20] [21]. For this reason, some studies select the ferromagnetic high-spin magnetic state of austenite which has a relaxed lattice parameter $a=3.64 \AA[22]$. Hydrogen-vacancy interactions in fcc iron have been investigated using both nonmagnetic (NM) and AFMD states; there were differences in the energy values for the two states but the qualitative trends were independent of the magnetic state [23]. Others have considered only the NM state in the assessment of carbon [24]. Bearing these studies in mind, we have assumed the non-magnetic state of austenite in the present work.

The lattice parameter of austenite was calculated to be $3.44 \AA$, which com- 
pares well with the range of values in published work for NM austenite, 3.44$3.45 \AA[22,23]$. The reference state for hydrogen is the $\mathrm{H}_{2}$ molecule, calculated by putting $\mathrm{H}_{2}$ in a cubic box with $10 \AA$ sides and carrying out a $\Gamma$-point calculation. Its bond length was obtained to be $0.75 \AA$, consistent with [23] and the experimental value of $0.74 \AA[25]$. The lattice constant for $\mathrm{Fe}_{31} \mathrm{Al}$ was found to be $3.46 \AA$ which is $0.6 \%$ larger than that of pure austenite.

The solution energy of hydrogen in $\mathrm{Fe}_{32}$ and $\mathrm{Fe}_{31} \mathrm{Al}$ can be studied to assess the interaction with aluminium with the hydrogen atoms located preferentially in the octahedral interstices $[23,29]$. For the Al containing system, we prepared $\mathrm{Fe}_{31} \mathrm{Al}$ because the $\mathrm{Al}$ concentration is about $1.5 \mathrm{wt} \%$ which is the previously reported concentration in the literature $[11,12]$. There are 3 possible configurations with $\mathrm{Fe}_{31} \mathrm{AlH}$ as shown in Fig. 1. The most stable configuration was found to be oct1 from the calculation. Thus, all the following Al containing systems had the oct1 configuration. The solution energies are defined as follows:

$$
\begin{gathered}
\Delta E_{s}\left(\mathrm{Fe}_{32} \mathrm{H}\right)=E\left(\mathrm{Fe}_{32} \mathrm{H}\right)-E\left(\mathrm{Fe}_{32}\right)-\frac{1}{2} E\left(\mathrm{H}_{2}\right), \\
\Delta E_{s}\left(\mathrm{Fe}_{31} \mathrm{AlH}\right)=E\left(\mathrm{Fe}_{31} \mathrm{AlH}\right)-E\left(\mathrm{Fe}_{31} \mathrm{Al}\right)-\frac{1}{2} E\left(\mathrm{H}_{2}\right)
\end{gathered}
$$

The obtained solution energies were 0.07 and $0.01 \mathrm{eV}$ for $\mathrm{Fe}_{32} \mathrm{H}$ and $\mathrm{Fe}_{31} \mathrm{AlH}$, respectively. The error in the solution enthalpy, $\pm 0.01 \mathrm{eV}$, was calculated as the absolute difference between the default result $\left(\mathrm{ENCUT}=400 \mathrm{eV}, N_{k}=7\right.$ ) and those with highest values of the varied input parameters. Tables 1 and 2 show the convergency test results with varying ENCUT and $k$-points, respectively. Thus, it was found that $\mathrm{Al}$ reduces the hydrogen solution energy. We can consider this energy difference as the binding energy of hydrogen near $\mathrm{Al}$. Then, the binding energy of hydrogen near the $\mathrm{Al}$ atom is obtained to be $0.06 \mathrm{eV} \equiv 5.8 \mathrm{~kJ} \mathrm{~mol}^{-1}$.

To understand why Al decreases the hydrogen solution energy, the distance between $\mathrm{Al}$ and the nearest $\mathrm{Fe}$ atoms was investigated. In pure iron, the distance between nearest atoms is $2.445 \AA$, and increases to $2.510 \AA$ in the aluminiumcontaining austenite along all directions. Fig. 2a shows the relaxed atomic structure for $\mathrm{Fe}_{31} \mathrm{Al}$. The blue and grey spheres represent $\mathrm{Al}$ and $\mathrm{Fe}$ atoms respectively. The red arrows mean the same distance, $2.510 \AA$. It is noteworthy that the distance between $\mathrm{Fe}$ atoms decreased even though the lattice parameter increased to $3.46 \AA$ due to the repulsion around the $\mathrm{Al}$ atom. Since the most stable position of hydrogen is oct1, which has the largest space, we can say that the reduction of hydrogen solution energy comes from the repulsion around the $\mathrm{Al}$ atom.

In addition, the effect of $\mathrm{Mn}$ was investigated with the $\mathrm{Fe}_{27} \mathrm{Mn}_{5}$ system con- 
sidering the high Mn concentration of TWIP steels. , Fig. 2b shows the atomic structure of the $\mathrm{Fe}_{26} \mathrm{Mn}_{5} \mathrm{AlH}$ system. The lattice parameters of $\mathrm{Fe}_{27} \mathrm{Mn}_{5}$ and $\mathrm{Fe}_{26} \mathrm{Mn}_{5} \mathrm{Al}$ were found to be 3.45 and $3.46 \AA$. The hydrogen solution energies of $\mathrm{Fe}_{27} \mathrm{Mn}_{5} \mathrm{H}$ and $\mathrm{Fe}_{26} \mathrm{Mn}_{5} \mathrm{AlH}$ were found to be 0.045 and $-0.019 \mathrm{eV}$ respectively. In this case, the binding energy due to the $\mathrm{Al}$ atom can be obtained as $6.1 \mathrm{~kJ} \mathrm{~mol}^{-1}$, which is almost the same as that of pure austenite. The solution energies and binding energies of $\mathrm{Al}$ are shown in Table 3. Calculations were done using different configurations of $\mathrm{Mn}$ atoms, and the results were not found to be significantly different.

A trap is defined as a location within the lattice where a hydrogen atom can enter a potential well that is deeper than in the perfect lattice. The first principles calculations indicate that the $\mathrm{Al}$ atom is such a trap; there are 8 possible positions for hydrogen in this supercell. If it is assumed that only half of those sites can be occupied at any instant due to repulsion between adjacent hydrogen atoms, then the trap density is $4 /(2 \times 3.46 \AA)^{3} \equiv 1.21 \times 10^{28} \mathrm{~m}^{-3}$. The hydrogen occupancy of these traps should be much less than 1, enabling the use of some standard theory for the apparent diffusivity $D$ as follows [27]:

$$
D=\frac{D_{L}}{1+\frac{N_{t}}{N_{l}} \exp \left(\frac{E_{b}}{R T}\right)}
$$

where $D_{L}$ is the hydrogen diffusivity in the austenite lattice in the absence of traps, $N_{l}$ and $N_{t}$ are the densities of lattice and trap sites, respectively, $R$ is the gas constant, $T$ is the absolute temperature, and $E_{b}$ is the binding energy. Furthermore, the amount of trapped hydrogen is given by:

$$
C_{t}=C_{l} \frac{N_{t}}{N_{l}} \exp \left(\frac{E_{b}}{R T}\right)
$$

where $C_{l}$ and $C_{t}$ are concentrations of hydrogen at lattice and trap sites. Fig. 3(a) compares the hydrogen diffusivity in austenite (304L stainless steel [28]) to the lower diffusivity obtained for the Al containing austenite. To the best of our knowledge, there is no report about the direct observation of the diffusivity of hydrogen in pure austenite or TWIP steels. It is emphasised that the reduction of diffusivity is due solely to the aluminium atoms acting as weak traps, and not due to any changes in the local atomic arrangements around the aluminium atom. Fig. 3(b) shows the ratio of total absorbed hydrogen calculated considering the Al trap effect using equation 3. Even though the $\mathrm{Al}-\mathrm{H}$ binding energy is relatively small, it can increase the total amount of absorbed hydrogen almost 2.3 times at room temperature. In practice, the degree of increase should be less than 2.3 because we didn't consider the trap effects from other trap sites such as dislocation or grain boundary. Besides, the measured hydrogen amount is depending on the penetration depth, which may differ from austenite to $\mathrm{Al}$ containing austenite due to the different diffusivity. Indeed, it was reported that the addition of $\mathrm{Al}$ increased the total amount of 
absorbed hydrogen by $20 \%[11,12]$.

The effect of $\mathrm{Al}$ addition on hydrogen thermal desorption with the obtained trap density and binding energy have been simulated as reported in detail in [26]. This simulation involves the lattice hydrogen diffusion and trapping or detrapping behaviour based on the local equilibrium assumption of Oriani [27]. The thermal desorption rates of austenite and Al-containing austenite were simulated assuming the following conditions: thickness $1 \mathrm{~mm}$, heating rate $100{ }^{\circ} \mathrm{C} \mathrm{h}^{-1}$, charging time $72 \mathrm{~h}$, and room temperature releasing time $30 \mathrm{~min}$. The diffusivity was obtained from [28], and the surface hydrogen concentration was 23 ppmw, as reported by [11]. This value for lattice diffusivity $D_{L}$ is used for both simulations, but when aluminium atoms are present, they act as traps and therefore the overall diffusivity in the simulation becomes $D$. The trap density and binding energy were obtained as discussed earlier in this paper. The predicted results are shown alongside published data $[11,12]$ in Fig. 4. The original measurements temperature $[11,12]$ monitored temperatures via the furnace controller; we therefore conducted calibration experiments by attaching a thermocouple to the sample itself, and relating the directly measured temperature to that output by the TDA equipment. The plots in Fig. 4 are therefore corrected relative to the originals. The specimen thickness is identical for all those results but the charging time of [12] is $48 \mathrm{~h}$ while the simulation and [11] used $72 \mathrm{~h}$. No significant change in the peak temperature with the addition of $\mathrm{Al}$ was predicted, but the peak height and total amount of hydrogen increased. These predictions coincide with the experimental observations.

To summarise, it is found that aluminium atoms in TWIP steel cause a localised dilation that better accommodates hydrogen, a phenomenon expressed via an Al-H binding energy. This binding energy when implemented in trapping theory indicates both that the presence of aluminium allows the TWIP steel to absorb more hydrogen than a corresponding steel that is aluminiumfree, and that the diffusion coefficient for hydrogen is significantly affected by the presence of aluminium.

Scripta Materialia 87 (2014) 9-12 


\section{References}

[1] L. Remy, A. Pineau: Materials science and engineering 28 (1977) 99-107.

[2] G. Frommeyer, U. Brüx, P. Neumann: ISIJ International 43 (2003) 438446.

[3] O. Bouaziz, O. S. Allain, C. Scott: Scripta Materialia 58 (2008) 484-487.

[4] J. A. Ronevich, S. K. Kim, J. G. Speer, D. K. Matlock: Scripta Materialia 66 (2012) 956-959.

[5] K. G. Chin, C. Y. Kang, S. Y. Shin, S. K. Hong, S. H. Lee, H. S. Kim, K. H. Kim, N. J. Kim: Materials Science \& Engineering A 528 (2011) 2922-2928.

[6] M. Koyama, E. Akiyama, T. Sawaguchi, D. Raabe, K. Tsuzaki: Scripta Materialia 66 (2012) 459-462.

[7] H.-J. Kim, S.-K. Youn: Journal of Manufacturing Science and Engineering, Transactions of the ASME 130 (2008) 0310051-0310057.

[8] M. Koyama, E. Akiyama, K. Tsuzak: ISIJ international 53 (2013) 12681274.

[9] I. Park, K. Jeong, J. Jung, C. Lee, Y. Lee: Int J Hydrogen Energ in press.

[10] Y. Chun, K. Park, C. Lee: Scripta Mater 66 (2012) 960-965.

[11] J. H. Ryu, S. K. Kim, C. S. Lee, D. W. Suh, H. K. D. H. Bhadeshia: Proceedings of the Royal Society A 469 (2013) 20120458.

[12] D. K. Han, Y. Kim, H. N. Han, H. K. D. H. Bhadeshia, D. W. Suh: Scripta Materialia 80 (2014) 9-12.

[13] Y. Li, C. Chen, F. Zhang: Advances in Materials Science and Engineering 2013 (2013) 382060.

[14] G. Cresse, J. Hafner: Physical Review B 47 (13115).

[15] G. Kresse, J. Furghmuller: Physical Review B 54 (1996) 11169.

[16] G. Kresse, J. Furghmuller: Computational Materials Science 6 (1966) 1550.

[17] J. P. Perdew, K. Burke, M. Ernzerhof: Physical Review Letters 77 (1996) 3865-3868.

[18] M. P. A. T. Methfessel, A. T. Paxton: Physical Review B 40 (1989) 36163621.

[19] H. C. Herper, E. Hoffmann, P. Entel: Physical Review B 60 (1999) 38393848.

[20] T. P. C. Klaver, D. J. Hepburn, G. J. Ackland: Physical Review B 85 (2012) 174111.

[21] M. Acet, H. Zähres, E. F. Wassermann, W. Pepperhoff: Physical Review B 49 (1994) 6012-6017.

[22] D. E. Jiang, E. A. Carter: Physical Review B 67 (2003) 214103.

[23] R. Nazarov, T. Hickel, J. Neugebauer: Physical Review B 82 (2010) 224104.

[24] A. Abbasi, A. Dick, T. Hickel, J. Neugebauer: Acta Materialia 59 (2011) 3041-3048.

[25] K. P. Huber, G. Herzberg: Constants of diatomic molecules, molecular 
spectra and molecular structure vol. iv (1979).

[26] E. J. Song, D. W. Suh, H. K. D. H. Bhadeshia: Computational Materials Science 79 (2013) 36-44.

[27] R. A. Oriani: Acta Metallurgica 18 (1970) 147-157.

[28] S. Xiukui, X. Jian, L. Yiyi: Materials Science \& Engineering A 114 (1989) 179-187.

[29] E. J. Song, H. K. D. H. Bhadeshia, D.-W. Suh: Corrosion Science 77 (2013) 379-384. 

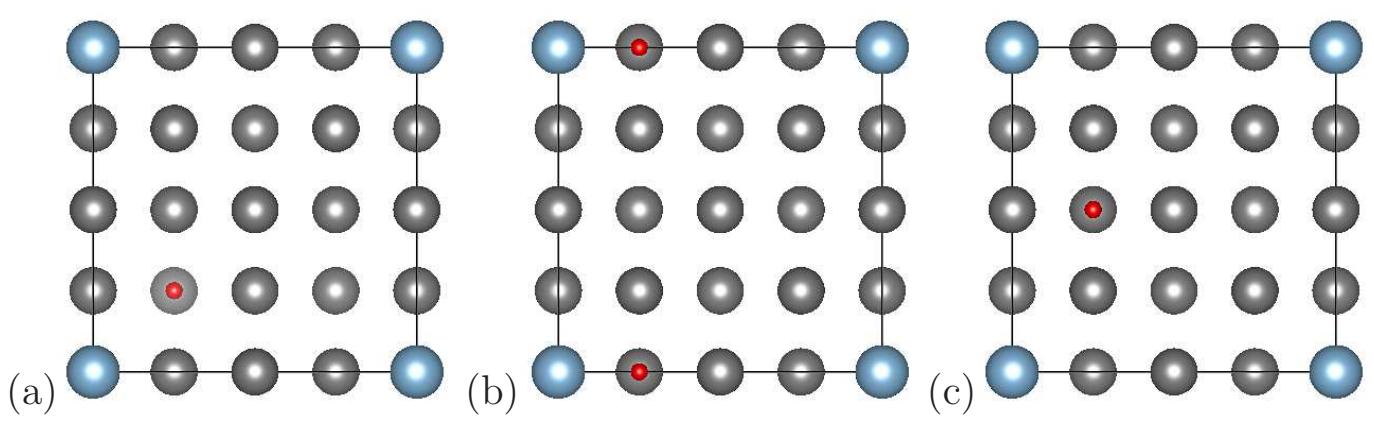

Fig. 1. Atomic structures of $\mathrm{Fe}_{31} \mathrm{AlH}$, (a) oct1, (b) oct2, and (c) oct3. The grey, blue, and red spheres represent $\mathrm{Fe}, \mathrm{Al}$, and $\mathrm{H}$ atom, respectively. The cell contains $31 \mathrm{Fe}$ atoms, $1 \mathrm{Al}$, and $1 \mathrm{H}$ atom.

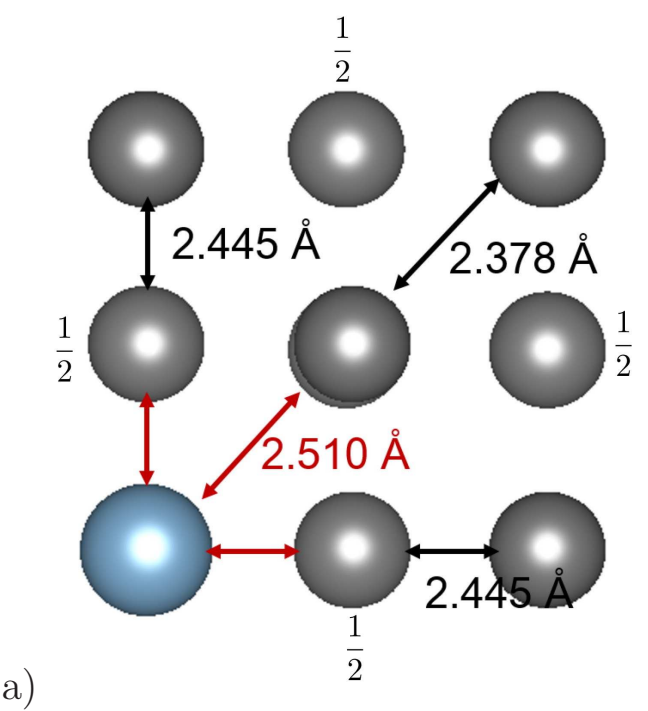

(b)

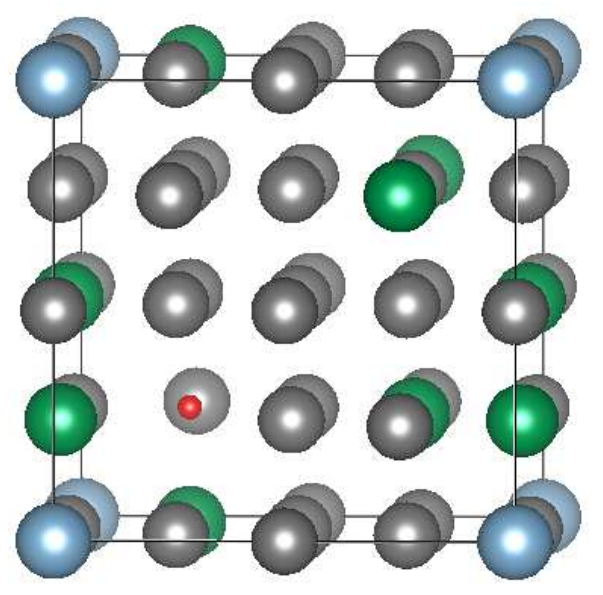

Fig. 2. (a) Atomic structure of $\mathrm{Fe}_{31} \mathrm{Al}$ with the distance between nearest atom. The red arrows indicate the same distance between $\mathrm{Al}$ and $\mathrm{Fe}$ atom. The fractions indicate the height of the atom in the direction normal to the diagram; unlabelled atoms are at heights 0,1 (b) Atomic structure of $\mathrm{Fe}_{26} \mathrm{Mn}_{5} \mathrm{AlH}$. The grey, green, blue and red spheres are $\mathrm{Fe}, \mathrm{Mn}, \mathrm{Al}$ and $\mathrm{H}$ atoms. Mn atoms were randomly added. 
(a)

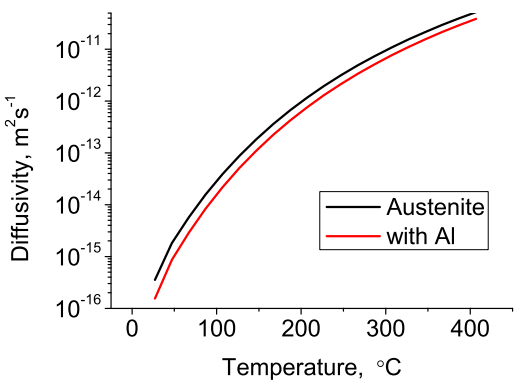

(b)

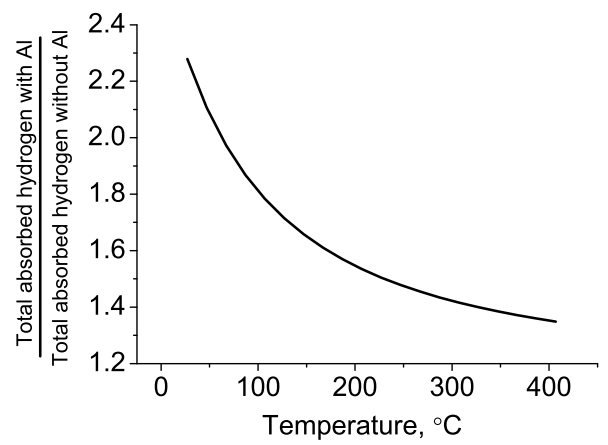

Fig. 3. The trap effect of $\mathrm{Al}$ on (a) hydrogen diffusivity (The black and red solid lines indicate the diffusivity of hydrogen in austenite and $\mathrm{Al}$ containing austenite, respectively) and (b) total absorbed hydrogen

Table 1

Convergence tests results of the energy per atom in bulk $\mathrm{Fe}_{32}, E_{\text {atom }}\left(\mathrm{Fe}_{32}\right), \mathrm{H}_{2}$ molecule, $E_{\text {atom }}\left(\mathrm{H}_{2}\right)$, and the solution energy of $\mathrm{Fe}_{32} \mathrm{H}, E_{s}\left(\mathrm{Fe}_{32} \mathrm{H}\right)$ with varying cutoff energy, ENCUT.

\begin{tabular}{cccccc}
\hline ENCUT & 200 & 300 & 400 & 500 & error \\
\hline$E_{\text {atom }}\left(\mathrm{Fe}_{32}\right), \mathrm{eV}$ & -8.222 & -8.153 & -8.144 & -8.144 & 0.000 \\
$E_{\text {atom }}\left(\mathrm{H}_{2}\right), \mathrm{eV}$ & -3.323 & -3.358 & -3.376 & -3.382 & 0.006 \\
$E_{s}\left(\mathrm{Fe}_{32} \mathrm{H}\right), \mathrm{eV}$ & 0.211 & 0.061 & 0.072 & 0.060 & 0.011 \\
\hline
\end{tabular}

Table 2

Convergence tests results of the energy per atom in bulk $\mathrm{Fe}_{32}, E_{\text {atom }}\left(\mathrm{Fe}_{32}\right)$, and the solution energy of $\mathrm{Fe}_{32} \mathrm{H}, \mathrm{E}_{s}\left(\mathrm{Fe}_{32} \mathrm{H}\right)$ with varying number of $k$-point, $N_{k}$.

\begin{tabular}{ccccc}
\hline$N_{k}$ & 5 & 7 & 9 & error \\
\hline$E_{\text {atom }}\left(\mathrm{Fe}_{32}\right), \mathrm{eV}$ & -8.144 & -8.144 & -8.144 & 0.000 \\
$E_{s}\left(\mathrm{Fe}_{32} \mathrm{H}\right), \mathrm{eV}$ & 0.087 & 0.072 & 0.077 & 0.005 \\
\hline
\end{tabular}

Table 3

The hydrogen solution energies and the binding energies of $\mathrm{Al}$ from first-principles calculations.

\begin{tabular}{ccccc}
\hline & $\mathrm{Fe}_{32} \mathrm{H}$ & $\mathrm{Fe}_{31} \mathrm{AlH}$ & $\mathrm{Fe}_{27} \mathrm{Mn}_{5} \mathrm{H}$ & $\mathrm{Fe}_{26} \mathrm{Mn}_{5} \mathrm{AlH}$ \\
\hline$E_{s}, \mathrm{eV}$ & 0.07 & 0.01 & 0.04 & -0.02 \\
$E_{b}, \mathrm{eV}$ & - & 0.06 & - & 0.06 \\
\hline
\end{tabular}




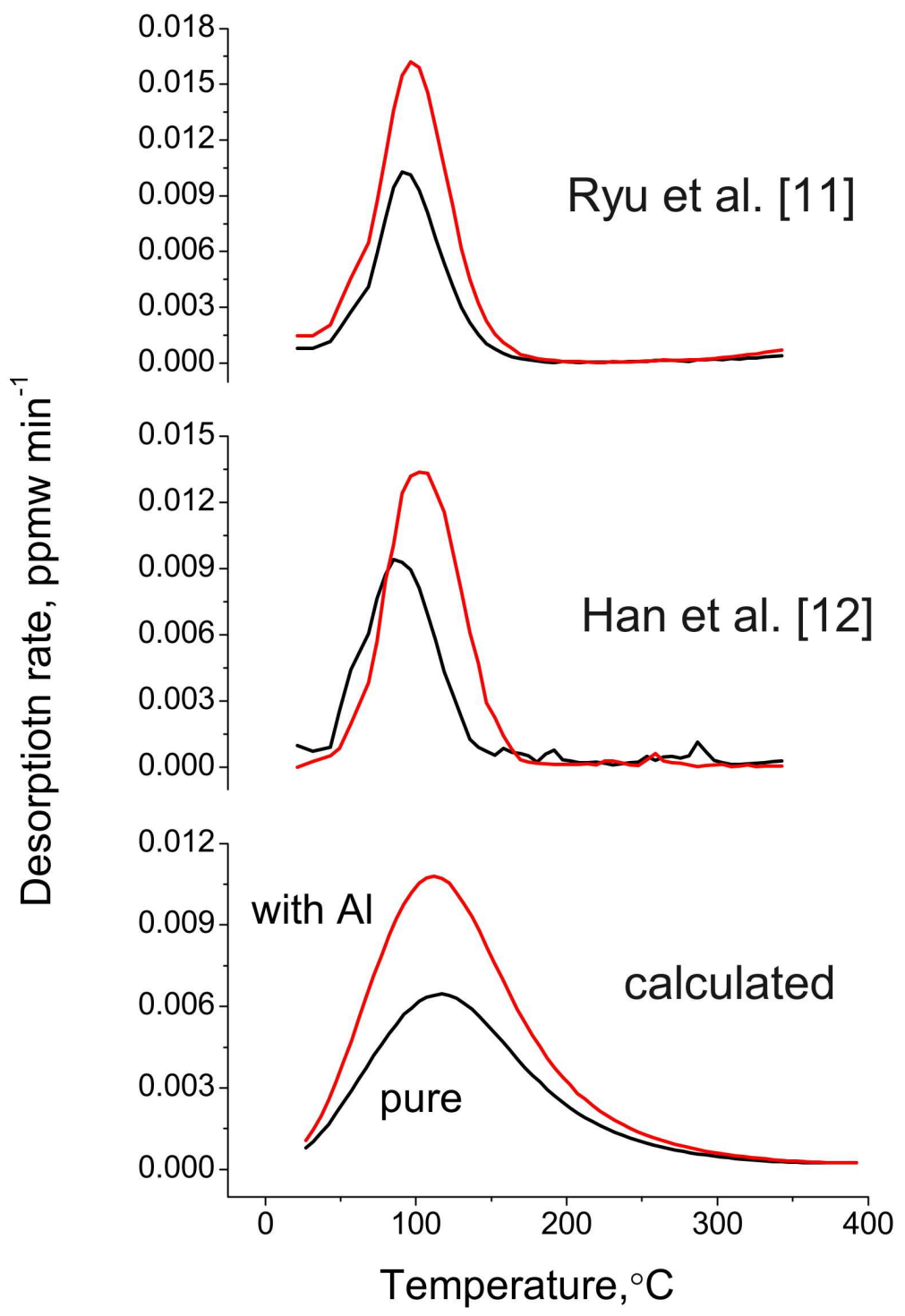

Fig. 4. The reported and predicted hydrogen thermal desorption rate of austenite and $\mathrm{Al}$ containing austenite with the calculated trap density and binding energy. The figures are reproduced from $[11,12]$ and the bottom figure is obtained by simulation. 